\title{
Cooperar en la diversidad es posible: Acuerdo de Bibliotecas Universitarias de Córdoba - Argentina
}

\author{
Por Sandra-Gisela Martín y Silvina-Marcela Angelozzi
}

\begin{abstract}
Resumen: Se presenta la experiencia de cooperación bibliotecaria de las universidades de la provincia de Córdoba, Argentina. Se enuncian las instituciones miembros del acuerdo, los objetivos, los productos y servicios consolidados y los que están en desarrollo. Finalmente se muestran los factores de éxito, los logros alcanzados y las dificultades encontradas.

Palabras clave: $A B U C$, Directorios de bibliotecas, Préstamo interbibliotecario, Catalogación cooperativa, Catálogos colectivos, Tesis y trabajos finales, Repositorios digitales.

Title: Cooperate in diversity is possible: experience of the Agreement of University Libraries of Córdoba - Argentina
\end{abstract}

Abstract: The experience of library cooperation among the universities of Argentina's Cordoba province is reported. The member institutions, objectives, products and services that are consolidated and those that are in development are described. Finally, the success factors, achievements and difficulties encountered are listed.

Keywords: $A B U C$, Library directories, Cooperative cataloguing, Union catalogue, Theses and dissertations, Digital repositories.

Martín, Sandra-Gisela; Angelozzi, Silvina-Marcela. “Cooperar en la diversidad es posible: Acuerdo de Bibliotecas Universitarias de Córdoba - Argentina". El profesional de la información, 2010, septiembre-octubre, v. 19, n. 5, pp. 514-518.

DOI: 10.3145/epi.2010.sep.11

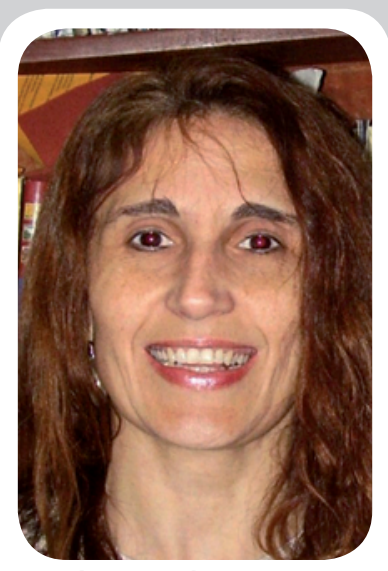

Sandra-Gisela Martín es licenciada en bibliotecología y documentación por la Univ. Nacional de Córdoba, Argentina, licenciada en informática por la Univ. Siglo XXl, y máster en documentación digital por la Univ. Pompeu Fabra. Directora del Sistema de Bibliotecas de la Universidad Católica de Córdoba y miembro de la Comisión Técnica de ABUC. Profesora adjunta de la Escuela de Bibliotecología de la Univ. Nacional de Córdoba, y de la licenciatura en bibliotecología a distancia de la Univ. Nacional de Litoral. Ha sido coordinadora de la red $A B U C$.

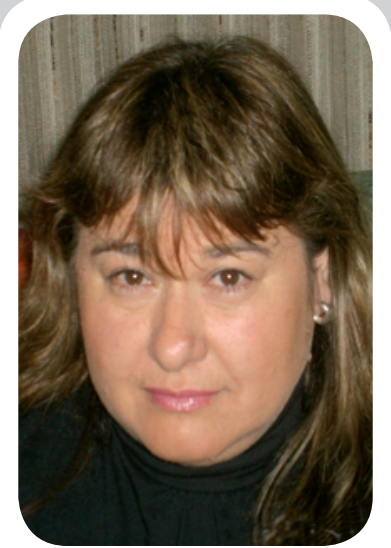

Silvina-Marcela Angelozzi es licenciada en bibliotecología y documentación por la Universidad Nacional de Córdoba, Argentina. Es directora de la Biblioteca Central de la Universidad Blas Pascal, y miembro de la Comisión Técnica de ABUC (Acuerdo de Bibliotecas Universitarias de Córdoba). Es profesora adjunta de la Escuela de Bibliotecología de la Universidad Nacional de Córdoba. Ha sido coordinadora de la red $A B \cup C$, ha dictado cursos, presentado ponencias en congresos y publicado artículos de la especialidad.

\section{Introducción}

EN LA PROVINCIA DE CÓRDOBA, Argentina, surgió en 2001 la primera experiencia de cooperación entre bibliotecas de instituciones académicas públicas $y$ privadas de la provincia.

Los entonces rectores de las universidades cordobesas reunidos en un Foro de Rectores firmaron un acuerdo marco que dio origen al Acuerdo de Bibliotecas Universitarias de Córdoba (ABUC).

http://www.abuc.org.ar

Está formado actualmente por las siguientes instituciones: $M i$ nisterio de Ciencia y Tecnología (provincia de Córdoba), Instituto
Universitario Aeronáutico, las universidades Blas Pascal, Católica de Córdoba, Empresarial Siglo 21, Nacional de Córdoba, Nacional de Río IV, Nacional de Villa María, Tecnológica Nacional Regional de Córdoba, Tecnológica Nacional Regional de San Francisco, y Tecnológica Nacional Regional de Villa María.

Este acuerdo tuvo un origen de arriba hacia abajo: los rectores acordaron la constitución del programa de cooperación en bibliotecas y a continuación los bibliotecarios documentalistas iniciaron el trabajo de organizar la red.

En base al camino recorrido de 2001 a 2010 podemos decir que el nacimiento como red cooperativa con esta estructura dio, entre otras, las siguientes ventajas: el apoyo claro y firme por parte de las autoridades; el convencimiento por parte de éstas de la necesidad de cooperar en materia de información; y el sentimiento de compartir entre las universidades cordobesas beneficios mutuos.

La misión de $A B U C$ es mejorar los servicios bibliotecarios a través de la cooperación y sus objetivos son:

- Gestionar el acceso a los catálogos de las bibliotecas.

- Incrementar la productividad científica y académica al mejorar el acceso de la comunidad universitaria a las colecciones existentes. 
- Mejorar los servicios bibliotecarios al compartir recursos.

- Promover planes de cooperación, servicios bibliotecarios conjuntos, adquisición compartida de recursos, y conexión a redes nacionales e internacionales.

- Experimentar y fomentar la aplicación de nuevas tecnologías de la información a los servicios bibliotecarios y potenciar la formación tecnológica del personal que trabaja en las bibliotecas.

- Colaborar con otras iniciativas de cooperación interbibliotecaria, especialmente en el ámbito de la provincia de Córdoba.

$\mathrm{Su}$ estructura está formada por un Consejo Coordinador (presidente, vicepresidente), un Coordinador ejecutivo del Programa, y una Comisión Técnica integrada por directores de bibliotecas y varias subcomisiones técnicas de estudio.

\section{Productos consolidados}

\section{Préstamo interbibliotecario (PI)}

El primer servicio que $A B U C$ implementó fue el préstamo interbibliotecario (PI) para fomentar el uso compartido de los fondos entre distintas unidades de información.

El procedimiento que se sigue es que el usuario debe realizar el pedido en la biblioteca a la cual pertenece, y el director de la institución y/o el responsable de PI del centro solicita el material a la biblioteca suministradora.

Ninguna biblioteca acepta peticiones provenientes directamente de los usuarios. El préstamo se realiza institucionalmente, por lo tanto el centro solicitante es responsable de la devolución oportuna del material.

\section{Catálogo colectivo de tesis y tra- bajos finales}

En Córdoba el Catálogo Colectivo de Tesis y Trabajos Finales de $A B U C$ es el primer catálogo colec- tivo referencial conformado por trabajos finales de carreras de grado y tesis de maestrías y doctorados pertenecientes a varias instituciones.

\section{http://www.abuc.org.ar/catalogo_} tesis/

$\mathrm{Su}$ objetivo general es contribuir a difundir y aumentar la visibilidad de la producción intelectual de estos trabajos. Los objetivos específicos son:

- difundir la producción académica e intelectual de cada una de las universidades;

- brindar a los usuarios información detallada sobre los distintos trabajos finales y tesis presentadas: temas, autores, grados académicos, etc.,

- permitir un mayor aprovechamiento de las colecciones a partir de una mayor difusión.

Se definieron los campos mínimos para cada registro de tesis: Tipo de tesis (trabajos finales, tesis de maestría y tesis de doctorado); Título; Autores; Director; Co-Director/Asesores; Título obtenido; Tribunal evaluador; Año; Fecha de defensa; Nota obtenida; Número de páginas; Descripción; Resumen; Palabras clave; Ubicación; Archivo de tesis; Comentarios internos; Fecha de alta; Ultima modificación; Estado (activo, desactivo).

El catálogo, que contiene ya 9.790 tesis, tiene estas características técnicas:

a) Módulo de administración central. Está integrado al sistema de administración del directorio de $A B U C$, lo cual es cómodo para administrar los ítems. Da acceso a todas las tesis, categorías, subcategorías, autores, instituciones, etc. El administrador del sistema es el encargado de dar de alta los nombres de usuarios y las claves de cada biblioteca. Tiene permiso para modificar, crear o eliminar los siguientes apartados:
- Regiones: Córdoba, San Francisco, Río Cuarto, Villa María.

- Redes de bibliotecas: ABUC, Vitruvio, Unired, etc.

- Instituciones: se registran aquí los nombres de las distintas instituciones.

- Directores: nombre y apellido de los responsables y/o directores de las bibliotecas.

- Administradores de cada biblioteca: el administrador general es el encargado de dar un nombre de usuario y una clave a cada persona encargada de la actualización de los datos de tesis en cada centro.

- Categorías de temas: por ejemplo, educación.

- Temáticas: educación física, educación ambiental...

- Tipos de bibliotecas: universitaria, académica, especializada.

- Bibliotecas: nombres y siglas de cada una.

- Contactos: mensajes de los lectores.

b) Módulo de búsquedas. Permite búsquedas básicas por tipo de tesis, institución y palabras; y búsquedas avanzadas por tipo de tesis, institución, palabras clave, autor, título y por año.

c) Integridad de interfaces gráficas con respecto al sistema de directorio. Se articula con los datos del Directorio de Bibliotecas Académicas y Científicas de Córdoba.

d) Tecnología. Ajax, basado en $P H P$ y $M Y S Q L$.

e) Actualización descentralizada. Cada biblioteca posee un "nombre de usuario" y una "clave" para actualizar sus registros. Sólo puede modificar sus propios registros.

Las principales ventajas de la administración descentralizada son que cada biblioteca es responsable de la actualización de sus registros de tesis; no hay que establecer me- 


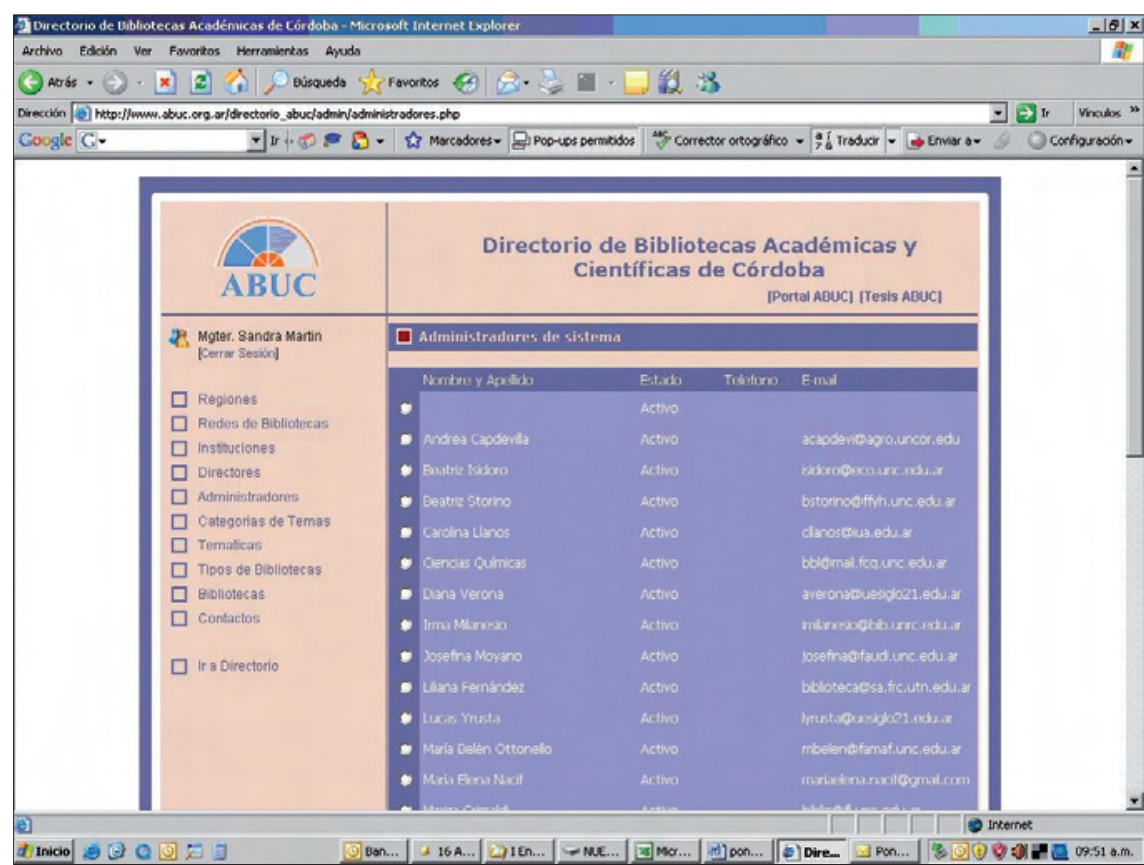

Figura 1. Catálogo Colectivo de Tesis y Trabajos Finales de ABUC

canismos de importación de registros para actualizar la base; y cada biblioteca puede hacer control de calidad de sus registros y realizar los cambios de manera inmediata.

Por el contrario, las principales desventajas son que cada centro debe cargar dos veces sus registros de tesis (una en su software local y otra en el catálogo colectivo); pueden generarse incoherencias si quienes administran no cumplen fielmente con las instrucciones de carga propuestas; y que problemas internos de las bibliotecas pueden hacer que no se actualice tan rápidamente como sería deseable.

f) Módulo de estadísticas. De acceso restringido para la Comisión Técnica de $A B U C$ a través de Google Analytics.

\section{http://www.google.com/analytics/}

Esta herramienta permite llevar un registro de consultas al sitio de $A B U C$ y en particular al catálogo. Se puede conocer la procedencia de los usuarios por país, la forma en que interactúan, la cantidad de consultas por período de tiempo, el porcentaje de nuevas visitas, idiomas, promedio de tiempo de permanencia de los visitantes, etc.

\section{Boletín electrónico Noticias de $A B U C$}

Destinado a difundir noticias relativas al Acuerdo e información de actualidad para bibliotecarios. No tiene una periodicidad fija.

Bolsa de trabajo, pasantías y becas

Servicio a través de la web destinado a publicar solicitudes y ofertas de trabajo, pasantías y becas que surjan en el área de la bibliotecología.

\section{Directorio de Bibliotecas Acadé- micas y Científicas de Córdoba}

Cuenta con la información relativa a 135 bibliotecas ubicadas en Córdoba capital y en la provincia. Incluye bibliotecas universitarias, académicas, científicas, especializadas, y centros de documentación.

Tiene como objetivos:

- brindar a los usuarios información detallada sobre bibliotecas, instituciones, redes, contactos, etc.

- conocer los centros que poseen colecciones de una determinada temática,

- permitir un mayor aprovechamiento de las colecciones a par- tir del conocimiento de los distintos acervos bibliográficos.

Los datos informativos que comprende el directorio son:

- Nombre de la biblioteca

- Siglas

- Dependencia

- Director/a

- Región (Córdoba, Villa María, Río Cuarto, San Francisco)

- Ciudad

- Ubicación

- Teléfono

- Fax

- Email

- Web

- Horario de atención

- Tipo de biblioteca: especializada, universitaria

- Redes asociadas

- Cobertura temática

- Colecciones: cantidad de volúmenes, de títulos de publicaciones periódicas, de otros materiales

- Foto: posibilidad de incorporar hasta 4 imágenes de cada biblioteca.

Entre las características más destacadas del directorio podemos mencionar:

- Módulo de búsquedas. Proporciona búsquedas básicas por palabras clave, y avanzadas por: redes, regiones, tipos de bibliotecas, temáticas o que contenga determinadas palabras.

- Integridad de interfaces gráficas con respecto al catálogo de tesis.

- Tecnología. Ajax, basado en $P H P$ y $M y S Q L$.

- Actualización centralizada. Cada biblioteca puede enviar sus datos a la Comisión Técnica de $A B U C$ para que sean actualizados en el directorio. 


\section{Eventos de capacitación}

A lo largo de estos nueve años se han desarrollado distintas instancias de capacitación destinadas a directivos y personal de bibliotecas. Algunas de las temáticas tratadas fueron la evaluación de bibliotecas universitarias, la planificación estratégica, la atención a usuarios en el marco de los procesos de comunicación, atención al público en bibliotecas, RDA (resource description and access), gestión del préstamo interbibliotecario, formulación y evaluación de proyectos para bibliotecas universitarias, metodología de la investigación en bibliotecología y documentación, catalogación de publicaciones seriadas con $\operatorname{marc} 21$ y Aacr $2 r$, estadísticas bibliotecarias para la generación de indicadores.

También se ha recibido la visita de colegas extranjeros de Estados Unidos y España, que transmitieron su experiencia a través de conferencias. Es de destacar que todos los eventos organizados han sido gratuitos para los bibliotecarios.

Asimismo, desde 2005 se realiza anualmente un encuentro bibliotecario de $A B U C$, abierto a todos los colegas.

\section{"Se ha roto con algunas dicotomías: bibliotecas grandes y pequeñas; bibliotecas con tradición de siglos y en formación; de gestión pública y de gestión privada"}

\section{Productos en desarrollo}

- Repositorio digital de investigaciones científicas

La dificultad para acceder al texto completo de las investigaciones científicas de la provincia de

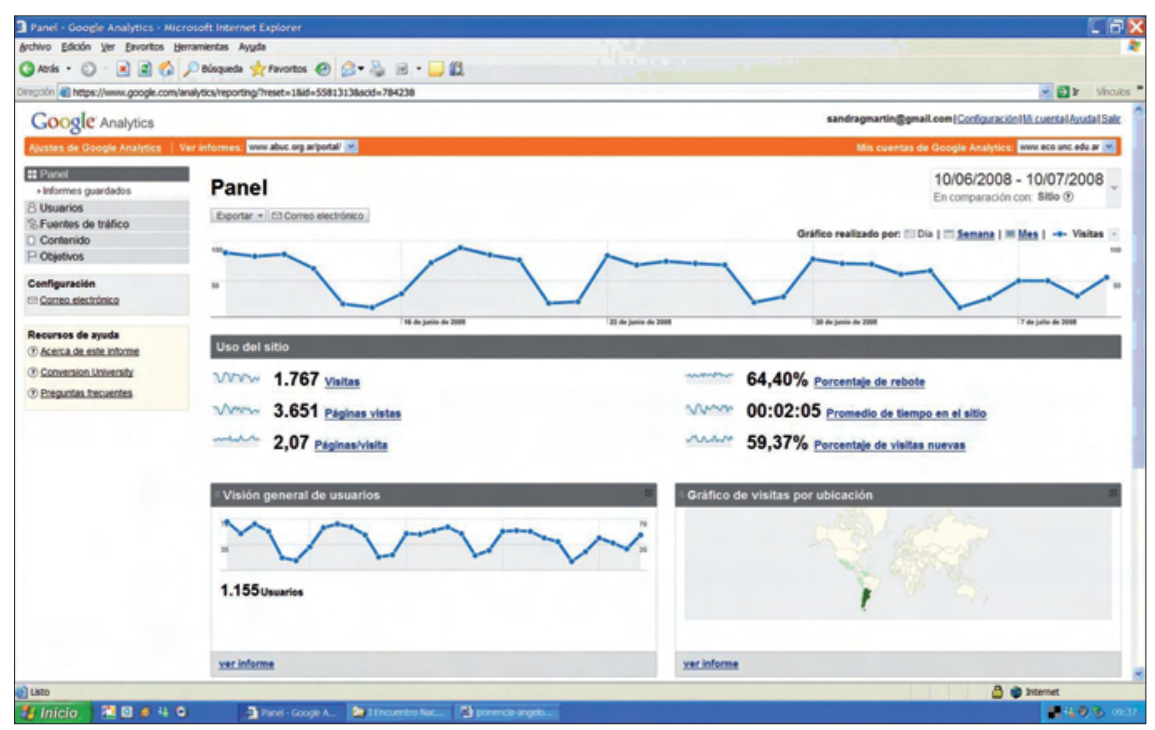

Figura 2. Utilización de Google Analytics para estadísticas

Córdoba es una problemática común para las bibliotecas de $A B U C$. A raíz de esta carencia surgió la idea de desarrollar un repositorio que reúna la producción de las instituciones participantes. El objetivo general es contribuir a difundir y aumentar la visibilidad de la producción intelectual de la provincia de Córdoba. Los objetivos específicos son:

- facilitar e impulsar la edición digital de documentos científicos y académicos;

- brindar un acceso único (catálogo colectivo digital en texto completo) a la documentación científica y académica de la provincia de Córdoba;

- favorecer la preservación;

- contribuir a la difusión de la producción científica cordobesa y guiar al establecimiento de políticas de investigación, evitando la duplicación de trabajos y haciendo más eficiente la producción científica de la provincia.

En Córdoba existen dos antecedentes importantes que dan origen a este proyecto: el Catálogo Colectivo de Tesis y Trabajos Finales ya mencionado, y la Biblioteca Digital del Ministerio de Ciencia y Tecnología de la Provincia de Córdoba. Esta biblioteca contiene una co- lección organizada de documentos científicos en formato digital que incluye servicios de búsqueda $\mathrm{y}$ recuperación de información. Se compone de dos bases de datos, una con proyectos de investigación de diferentes disciplinas financiados por el Gobierno de la provincia de Córdoba desde 1994, y la segunda con los productos que reflejan los resultados de las investigaciones: publicaciones, patentes, ponencias, videos, etc. La Biblioteca ofrece los datos de localización de dichos productos y copia de la mayoría de ellos en soporte papel.

El repositorio de acceso abierto contendrá la producción científica y tecnológica de cada una de las instituciones miembros, que en estos momentos es de unas 3.000 tesis de maestría y doctorales y unos 6.600 trabajos de investigación del período 2004-2009.

- Software para automatización de transacciones de préstamos interbibliotecarios

Actualmente se encuentra en desarrollo un sistema de gestión de préstamos interbibliotecarios en entorno web, que permitirá, entre otras, las funciones de administración, seguimiento y logística del préstamo, y reportes estadísticos.

- Sistema de gestión e intercambio de ejemplares duplicados. 


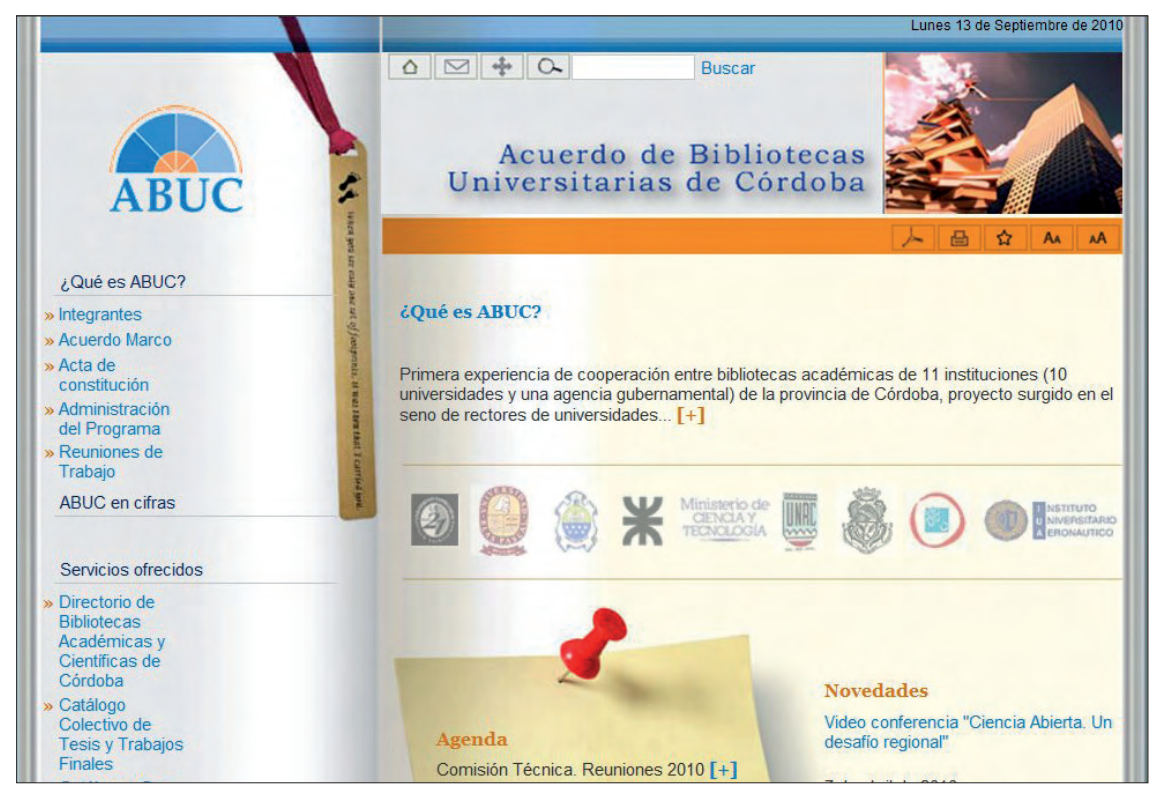

http://www.abuc.org.ar

Este sistema permitirá localizar material duplicado entre bibliotecas con el objeto de ayudar a completar colecciones o a ubicar material no disponible para la venta.

\section{"Aunque el crecimiento de productos y servicios se realiza lentamente, los logros alcanzados han beneficiado a todas las instituciones participantes"}

\section{Conclusiones}

Existen ciertos factores que han contribuido a que la red permanezca en el tiempo, como: el apoyo firme de parte de las autoridades, la buena difusión, una buena comunicación entre los participantes, procesos de decisión consensuados, reuniones de trabajo basadas en el respeto mutuo, y una actitud proactiva.

En los nueve años de existencia del acuerdo podemos señalar como logros principales:

- las bibliotecas de universidades pequeñas y en formación tienen un mejor posicionamiento y mayor visibilidad;
- las colecciones de cada biblioteca se han visto ampliadas y complementadas con los fondos bibliográficos de las demás instituciones al compartir el acervo;

- a los usuarios se les ofrece servicio de préstamo interbibliotecario;

- se realizan eventos de capacitación conjuntos compartiendo los gastos, que han beneficiado a numerosos agentes de las diferentes bibliotecas;

- mejor posición ante las autoridades universitarias;

- se favorece la integración del personal de las bibliotecas a través de eventos compartidos como jornadas, cursos y seminarios;

- se ha roto con algunas dicotomías: bibliotecas grandes y pequeñas; bibliotecas con tradición de siglos y en formación; de gestión pública y de gestión privada;

- se ha demostrado que es posible la cooperación en la diversidad.

A lo largo de este camino también aparecieron dificultades a superar:

- Las diferencias de gestión de las distintas universidades hacen que los tiempos y recursos de cada una sean diferentes, lo cual hace difícil en ocasiones coordinar las actividades.

- El prejuicio existente en la sociedad y en los mismos bibliotecarios entre universidades de gestión pública y de gestión privada ha generado roces en ciertas ocasiones.

- La no participación durante algunos períodos de tiempo de algunos integrantes, motivados fundamentalmente por la carencia de recursos económicos.

- Desigual compromiso entre los participantes.

- Para los representantes de las bibliotecas ante $A B U C$, el trabajo para el acuerdo se suma a las tareas habituales, por lo que en muchas ocasiones se dilatan los tiempos previstos para la culminación de los proyectos.

Haciendo un balance general podemos concluir que los logros de $A B U C$ superan ampliamente las dificultades y a pesar de que el crecimiento de productos y servicios se realiza lentamente, los logros alcanzados han beneficiado a todas las instituciones participantes.

\section{Bibliografia}

Acta de Constitución. Acuerdo de Bibliotecas Universitarias de Córdoba, 2001.

http://www.abuc.org.ar/portal/?sec $=2 \&$ pag $=$ 21\#lectura

Acuerdo de Bibliotecas Universitarias de Córdoba $(A B U C), 2010$.

http://www.abuc.org.ar

Acuerdo Marco [Bibliotecas Universitarias de Córdoba], 2001.

http://www.abuc.org.ar/portal/?sec $=2 \&$ pag $=$ 20\#lectura

Google analytics, 2010.

http://www.google.com/analytics/

Sandra-Gisela Martín. Universidad Católica de Córdoba, Argentina. sandragmartin@gmail.com

Silvina-Marcela Angelozzi. Universidad Nacional de Córdoba, Argentina.

smangelozzi@gmail.com 